Artigo

\title{
Doença arterial periférica e diabetes mellitus tipo 2: uma comparação entre a epidemiologia e o manejo brasileiro e austríaco
}

\author{
Catarina Kim Masukawa de Souza ${ }^{1}$
}

1. Estudante do Curso de Graduação em Medicina, Centro de Ciências da Saúde, Universidade Federal de Santa Catarina (UFSC), Campus Reitor João David Ferreira Lima, Florianópolis, Brasil.

\section{RESUMO}

Breve revisão comparativa entre os protocolos brasileiro e austríaco para prevenção, diagnóstico e tratamento da doença arterial periférica no diabetes mellitus tipo 2.

DOI: https://doi.org/10.32963/bcmufsc.v6i1.3870 Palavras-chave: Diabetes Mellitus; Diabetes Mellitus tipo 2; Doença Arterial Periférica; Protocolo Submetido em 18/12/2019; aceito para publicação em 29/05/2020. A autora declara não haver conflitos de interesse na formulação desse artigo. Autor para contato: Catarina Kim Masukawa de Souza. E-mail: catarinakin@gmail.com

\begin{abstract}
A tes de 2008, dados epidemiológicos sobre Doença Arterial Periférica (DAP) no Brasil eram escassos e limitados a populações específicas. Quatro anos antes disso, um grupo de médicos de São Paulo iniciou um estudo transversal, multicêntrico e nacional com 1.170 indivíduos de 72 grandes centros urbanos brasileiros ${ }^{1}$, buscando estabelecer a prevalência da doença no país, bem como os principais fatores de risco associados à ela. Os resultados desse estudos mostraram uma prevalência de DAP na população geral ( $>18$ de anos) de 10,5\% e estabeleceram a diabetes mellitus tipo 2 (DMT2) como o principal fator de risco. Esses resultados serviram de grande alerta à comunidade médica brasileira sobre a necessidade urgente de se rastrear a DAP na prática clínica, especialmente entre pacientes diabéticos assintomáticos. Os diabéticos, especificamente no Brasil, correspondem a 7,5\% da população geral - de acordo com um estudo de $2017^{2}-$ o que significa aproximadamente 3,5 milhões de pessoas. Foi observado, também nesse mesmo estudo, que em pacientes com DAP, a prevalência de diabetes era quase três vezes maior quando comparada a grupos sem DAP 1.
\end{abstract}

Foi apenas em 2015 que a Sociedade Brasileira de Diabetes publicou uma pesquisa em relação ao manejo de diabéticos com DAP $\mathrm{DP}^{3}$. O objetivo era estabelecer uma conduta mais clara que atendesse as particularidades desses pacientes, que geralmente evoluem com casos mais severos da doença. Nesse estudo é colocado que pacientes diabéticos (entre 50-59 anos) assintomáticos para DAP e com outros fatores de risco, pacientes diabéticos sintomáticos para DAP e pacientes diabéticos

Bol Curso Med UFSC 2020; 6 (1) com idade maior que 70 anos deveriam ser submetidos à triagens por meio do Índice Tornozelo-Braquial (ITB). Não fica claro, no entanto, a frequência com que esse exame complementar deve ser feito em pacientes assintomáticos. A pesquisa também ressalta que exames de imagem não devem ser realizados com função diagnóstica, mas sim quando intervenções - como a revascularização - são consideradas para opção terapêutica. Ainda, a mesma publicação afirma que a conduta terapêutica é baseada em dois pilares: controlar os fatores de risco e tratar os sintomas da isquemia periférica. $\mathrm{O}$ primeiro pilar deve ser alcançado por, primeiramente, cessação do tabagismo, controle glicêmico $(\mathrm{HbA} 1 \mathrm{c}<7 \%)$, controle da hipertensão (níveis pressóricos $<130 \times 80 \mathrm{mmHg}$ ), controle da hiperlipidemia (LDL $\quad<100 \mathrm{mg} / \mathrm{dL}$ ou $<70 \mathrm{mg} / \mathrm{dL}$ se antecedente aterosclerótico), e, por fim, terapia antiplaquetária com clopidogrel ou ácido acetilsalicílico (AAS). Com relação ao tratamento clínico dos sintomas, o protocolo sugere que, em caso de claudicação intermitente, a atividade física é a principal medida terapêutica. Seguindo a aprovação pelo FDA, terapia farmacológica com cilostazol (vasodilatador e antiagregante plaquetário) também é recomendada - exceto em paciente com insuficiência cardíaca congestiva e disfunções hepáticas ou renais. Em casos nos quais o manejo clínico é insuficiente, ou casos severos (III e IV na classificação de Fontaine), a intervenção cirúrgica é indicada. No Brasil, cirurgia aberta (bypass) ainda é o procedimento de escolha, apesar do crescente uso de procedimentos endovasculares. A escolha entre essas duas técnicas reside na localização do território anatômico acometido. 
Quanto aos protocolos austríacos, que seguem as orientações formuladas em 2019 pela Sociedade Europeia de Cardiologia/Associação Europeia pelo Estudo da Diabetes ${ }^{4}$, o tratamento é baseado, primeiramente, em mudanças no estilo de vida e cessação imediata do tabagismo; uso de drogas para controle dislipidêmico, sendo estatinas a primeira escolha e o LDL almejado $<70 \mathrm{mg} / \mathrm{dL}$ (ezetimibe também pode ser usado em situações especiais) e drogas anti-hipertensivas que mantenham os níveis pressóricos <140x85 mmHg. Quanto à terapia anti agregação plaquetária é colocado que:

- $\quad$ Em pacientes com DM e risco cardiovascular (CV) moderado, terapia primária para prevenção de DAP não é recomendada;

- Somente pacientes diabéticos com risco alto/muito alto para eventos $\mathrm{CV}$, assintomáticos para DAP, devem receber AAS;

- Terapia dupla (AAS + clopidogrel) é recomendada apenas para pacientes diabéticos com DAP sintomática, e não como prevenção primária;

- $\quad$ Cilostazol não é recomendado.

Comparando ambos os protocolos, os parâmetros brasileiros e austríacos diferem, sendo os brasileiros um pouco mais extremos, principalmente quanto ao controle da hipertensão, a prevenção primária e o uso de cilostazol. A hipertensão, pelos guidelines europeus, é mais tolerada, inclusive sugerindo que uma baixa pressão arterial média não significa uma melhora em prognóstico. Quanto à prevenção primária, a principal diferença reside no uso de anti-agregantes plaquetários: no Brasil, DMT2 por si só sugere o uso de asprina ou clopidogrel para prevenir DAP. O mesmo não se aplica às diretrizes europeias, que utilizam aspirina apenas em casos de risco alto/muito alto de eventos CV. Entretanto, o tratamento para DAP sintomática é igual em ambos os protocolos: aspirina + clopidogrel. E, apesar de aprovação pelo FDA, os protocolos europeus não recomendam o uso de cilostazol para o tratamento de DAP. Outra divergência reside na intervenção cirúrgica: na Áustria, a primeira escolha sempre é endovascular com angioplastia periférica, sendo bypass uma segunda opção.

Em conclusão, de várias maneiras o Brasil e a Áustria seguem práticas similares no manejo da DAP. Entretanto, há diferenças, sendo os protocolos brasileiros mais baseado nos norte americanos do que nos europeus. É necessária uma revisão geral a respeito da interação entre DMT2 e da DAP, adaptados à realidade brasileira, a medida que novos guidelines estrangeiros surjam.

\section{Referências}

1. Makdisse, M., Pereira, A. D. C., Brasil, D. P., et al. Prevalência e fatores de risco associados à doença arterial periférica no Projeto Corações do Brasil. Arq Bras Cardiol 2008; 91:402-14

2. Flor, L. S., \& Campos, M. R. Prevalência de diabetes mellitus e fatores associados na população adulta brasileira: evidências de um inquérito de base populacional. Revista Brasileira de Epidemiologia 2017; 20, 16-29.

3. Sociedade Brasileira de Diabetes. Doença arterial obstrutiva periférica no paciente diabético: avaliação e conduta. Diretrizes SBD. 2015; $296-303$.

4. Cosentino, F., Grant, P. J., Aboyans, et al. (2020). 2019 ESC guidelines on diabetes, pre-diabetes, and cardiovascular diseases developed in collaboration with the EASD: the task force for diabetes, pre-diabetes, and cardiovascular diseases of the European Society of cardiology (ESC) and the European association for the study of diabetes (EASD). Eur Heart J 2020; 41: 255-323. 